\title{
FERMI/LAT STUDY OF GAMMA-RAY EMISSION IN THE DIRECTION OF THE MONOCEROS LOOP SUPERNOVA REMNANT
}

\author{
H. Katagiri ${ }^{1,10}$, S. Sugiyama ${ }^{1,10}$, M. Ackermann ${ }^{2}$, J. Ballet ${ }^{3}$, J. M. Casandjian ${ }^{3}$, Y. Hanabata ${ }^{4}$, J. W. Hewitt ${ }^{5}$, \\ M. KERR ${ }^{6}$, H. Kubo ${ }^{7}$, M. Lemoine-Goumard ${ }^{8}$, AND P. S. RaY ${ }^{9}$ \\ ${ }^{1}$ College of Science, Ibaraki University, 2-1-1 Bunkyo, Mito, Ibaraki 310-8512, Japan; hideaki.katagiri.sci@vc.ibaraki.ac.jp \\ ${ }^{2}$ Deutsches Elektronen Synchrotron DESY, D-15738 Zeuthen, Germany \\ ${ }^{3}$ Laboratoire AIM, CEA-IRFU/CNRS/Université Paris Diderot, Service d'Astrophysique, CEA Saclay, F-91191 Gif sur Yvette, France \\ ${ }^{4}$ Institute for Cosmic-Ray Research, University of Tokyo, 5-1-5 Kashiwanoha, Kashiwa, Chiba 277-8582, Japan \\ ${ }^{5}$ University of North Florida, Department of Physics, 1 UNF Drive, Jacksonville, FL 32224, USA \\ ${ }^{6}$ CSIRO Astronomy and Space Science, Australia Telescope National Facility, Epping NSW 1710, Australia \\ ${ }^{7}$ Department of Physics, Graduate School of Science, Kyoto University, Kyoto, Japan \\ ${ }^{8}$ Centre d'Études Nucléaires de Bordeaux Gradignan, IN2P3/CNRS, Université Bordeaux 1, BP120, F-33175 Gradignan Cedex, France \\ ${ }^{9}$ Space Science Division, Naval Research Laboratory, Washington, DC 20375-5352, USA \\ Received 2016 June 10; revised 2016 August 13; accepted 2016 August 21; published 2016 October 31
}

\begin{abstract}
We present an analysis of the gamma-ray measurements by the Large Area Telescope on board the Fermi Gammaray Space Telescope in the region of the supernova remnant (SNR) Monoceros Loop (G205.5+0.5). The brightest gamma-ray peak is spatially correlated with the Rosette Nebula, which is a molecular cloud complex adjacent to the southeast edge of the SNR. After subtraction of this emission by spatial modeling, the gamma-ray emission from the SNR emerges, which is extended and fit by a Gaussian spatial template. The gamma-ray spectra are significantly better reproduced by a curved shape than a simple power law. The luminosities between 0.2 and $300 \mathrm{GeV}$ are $\sim 4 \times 10^{34} \mathrm{erg} \mathrm{s}^{-1}$ for the SNR and $\sim 3 \times 10^{34} \mathrm{erg} \mathrm{s}^{-1}$ for the Rosette Nebula, respectively. We argue that the gamma-rays likely originate from the interactions of particles accelerated in the SNR. The decay of neutral pions produced in nucleon-nucleon interactions of accelerated hadrons with interstellar gas provides a reasonable explanation for the gamma-ray emission of both the Rosette Nebula and the Monoceros SNR.
\end{abstract}

Key words: acceleration of particles - cosmic rays - gamma-rays: ISM - ISM: individual objects (the Monoceros Loop) - ISM: supernova remnants

\section{INTRODUCTION}

The shock waves of supernovae accelerate particles to very high energies through the mechanism of diffusive shock acceleration (e.g., Blandford \& Eichler 1987). However, the processes of acceleration, release from the shock region, and diffusion in the interstellar medium of such particles are not well understood. Gamma-ray observations in the $\mathrm{GeV}$ domain are a powerful probe of these mechanisms. The Large Area Telescope (LAT) on board the Fermi Gamma-ray Space Telescope has detected $\mathrm{GeV}$ gamma-rays from several supernova remnants (SNRs; e.g., Thompson et al. 2012; Acero et al. 2016b, and references therein).

The Monoceros Loop (G205.5+0.5) is a well-studied middle-aged SNR. It has a large diameter $\left(\sim 3^{\circ} .8\right)$ that allows detailed morphological studies in high-energy gamma-rays since the LAT has a comparable point-spread function (PSF) above a few hundred $\mathrm{MeV}$ (the $68 \%$ containment angle above $1 \mathrm{GeV}$ is smaller than $1^{\circ}$ ). The radio emission of the SNR has a nonthermal spectrum (e.g., Xiao \& Zhu 2012), indicating the existence of high-energy electrons. A young stellar cluster and molecular cloud complex, the Rosette Nebula, is located at the edge of the southern shell of the SNR. The $\mathrm{H} \alpha$ line widths in the SNR ridge overlapping with the Rosette region are larger than near the center of the Rosette Nebula (Fountain et al. 1979), suggesting that the SNR is interacting with the Rosette Nebula. Turner (1976) obtained distances of $1.6 \mathrm{kpc}$ for stars associated with the Rosette Nebula from main-sequence fitting. Odegard (1986) argued that the distance of the SNR is

$\overline{10}$ Author to whom any correspondence should be addressed.
$1.6 \mathrm{kpc}$ based on his decameter wavelength observations of the absorption of nonthermal emission from the SNR by the Rosette Nebula. In this paper, we adopt this distance of $1.6 \mathrm{kpc}$ for both objects. The age was estimated to be $\sim 3 \times 10^{4}$ years based on the X-ray data and an SNR model (Leahy et al. 1986).

In Monoceros, a very-high-energy (VHE) gamma-ray source, HESS J0632+057, was first discovered at TeV energies by the High Energy Stereoscopic System (H.E.S.S.; Aharonian et al. 2007), located close to the rim of the Monoceros SNR. It appears to be point-like within experimental resolution; the limit on the size of the emission region was given as $2^{\prime}$ (a 95\% confidence level). Detection of variability in the VHE gammaray and X-ray fluxes supports interpretation of the object as a gamma-ray emitting binary (Acciari et al. 2009), indicating that the bulk of VHE gamma-rays do not come from high-energy particles accelerated by the SNR. No significant emission from the location of HESS J0632+057 was detected in the $0.1-100 \mathrm{GeV}$ energy range integrating over 3.5 years of Fermi/LAT data (Caliandro et al. 2013). Also, an unidentified high-energy gamma-ray source, 3EG J0634+0521, has been detected using the EGRET data (Hartman et al. 1999). However, morphological studies, which could associate the gamma-ray emission with molecular clouds require higher photon statistics with better angular resolution.

Seven LAT point-like sources positionally associated with the Monoceros Loop SNR (2FGL J0631.6+0640, 2FGL J0633.7+ 0633, 2FGL J0636.0+0554, and 2FGL J0637.8+0737) and the Rosette Nebula (2FGL J0631.7+0428， 2FGL J0634.3 $+0356 \mathrm{c}$, and 2FGL J0637.0+0416c) are listed in the 2FGL catalog (Nolan et al. 2012). However, the two sources 
(2FGL J0634.3+0356c and 2FGL J0637.0+0416c) associated with the Rosette Nebula were classified as "c" sources that require caution in interpreting or analyzing. In addition, the extended emission around the SNR was reported in the first Fermi/LAT SNR catalog (Acero et al. 2016b), which was modeled as a uniform disk with the radius of $2^{\circ} .3$. In order to understand the emission and its mechanism more deeply, a detailed analysis and further discussion are required.

In this paper, we report a detailed study of the emission in the direction of the Monoceros Loop by using the Fermi/LAT data. We have analyzed the 67-month LAT data by using the 2FGL catalog. Observations and data selection are briefly described in Section 2. The analysis procedure and results described in Section 3 include a study of the morphology and spectrum of the emission associated with the Monoceros Loop and the Rosette Nebula. Finally, we present our results in Section 4 and conclusions in Section 5. The data selection, analysis procedure, and the modeling of gamma-ray emission are based on the previous studies of the Cygnus Loop by Katagiri et al. (2011) and SNR HB 3 by Katagiri et al. (2016).

\section{OBSERVATIONS AND DATA SELECTION}

The main instrument on Fermi is the LAT, which detects gamma-rays from $\sim 20 \mathrm{MeV}$ to $>300 \mathrm{GeV} .{ }^{11}$ The LAT is an electron-positron pair production telescope, using tungsten foil converters and silicon microstrip detectors and a hodoscopic cesium iodide calorimeter to measure the arrival directions and energies of incoming gamma-rays. They are surrounded by 89 segmented plastic scintillators that serve as an anticoincidence detector to reject events originating from charged particles. Detailed information about the instrument can be found in Atwood et al. (2009), the on-orbit calibration is described in Abdo et al. (2009), and a summary of event classification strategies and instrument performance is given in Ackermann et al. (2012). The LAT has a larger field of view ( 2.4 sr), a larger effective area $\left(\sim 8000 \mathrm{~cm}^{2}\right.$ for $>1 \mathrm{GeV}$ on-axis peak effective area) and improved PSF in comparison to previous high-energy gamma-ray telescopes.

We analyzed events toward the Monoceros Loop recorded from the start of science operations on 2008 August 4 until 2014 January 29 . The LAT operated in a nearly continuous sky survey mode, to obtain a total exposure of $\sim 1.5 \times 10^{11} \mathrm{~cm}^{2} \mathrm{~s}$ (at $1 \mathrm{GeV})$. In this observing mode, approximately uniform coverage of the entire sky is obtained every two orbits ( $\sim 3 \mathrm{hr})$.

We used the standard LAT analysis software, the ScienceTools version v9r32, publicly available from the Fermi Science Support Center (FSSC). ${ }^{12}$ We use events classified as P7SOURCE that have been reprocessed with an updated instrument calibration (Bregeon et al. 2013). Only events that have a reconstructed zenith angle less than $100^{\circ}$ were used in order to minimize the contamination from Earth-limb gamma-ray emission. Furthermore, only time intervals when the center of the LAT field of view is within $52^{\circ}$ of the local zenith are accepted to further reduce the contamination by Earth's atmospheric emission. The Instrument response functions (IRFs) that correspond to this data set are P7REP_SOURCE_V15 (publicly available via the FSSC) throughout this work.

\footnotetext{
${ }_{11}$ Only events with energies $>0.2 \mathrm{GeV}$ are used in this analysis.

12 Software and documentation of the Fermi ScienceTools are distributed by the Fermi Science Support Center at http://fermi.gsfc.nasa.gov/ssc.
}

Times when the LAT detected a gamma-ray burst (GRB) or nova were eliminated from the data set. The transients located within $15^{\circ}$ of the Monoceros Loop were GRB 130504C (Kocevski et al. 2013) and Nova Mon 2012 (Cheung et al. 2013), corresponding to 56416.97797-56417.00390 and 56099.00000-56109.00000 in Modified Julian Day, respectively.

The region around the SNR is dominated by the gamma-ray emission of PSR J0633+0632 (2FGL J0633.7+0633). The pulse profile in the $0.2-300 \mathrm{GeV}$ energy range analyzed in this paper is shown in Figure 1, where the events are within $1^{\circ}$ of the pulsar position. Using a timing solution modeling the effects of spin-down and timing noise (Kerr et al. 2015), we assigned a rotational phase to each photon using the Fermi plug-in of the TEMPO2 software package (Hobbs et al. 2006). We only used the events during the off-pulse phases of PSR J0633+0632, corresponding to phases of 0.24-0.52 and 0.67-1.00 as adopted in Abdo et al. (2013). We restricted the energy range to $>0.2 \mathrm{GeV}$ to avoid possible large systematics due to the rapidly varying effective area and much broader PSF at lower energies.

\section{ANALYSIS AND RESULTS}

\subsection{General Settings}

The morphology and spectrum of gamma-ray emission from the Monoceros Loop and Rosette Nebula were determined using a binned likelihood analysis based on Poisson statistics $^{13}$ (see, e.g., Mattox et al. 1996). The likelihood is the product of the probabilities of the observed gamma-ray counts within each spatial and spectral bin for a specified model. The gamma-ray emission model used here included all sources detected in the 2FGL catalog within $20^{\circ}$ of the SNR. We also included the standard LAT diffuse background model (Acero et al. 2016a), gll_iem_v05_rev1.fit that results from cosmic-ray $(\mathrm{CR})$ interactions with the interstellar medium and radiation fields and an isotropic component to represent extragalactic gamma-rays and charged particle background using a tabulated spectrum (iso_source_v0 5 . txt). Both diffuse models are available from the FSSC. We fit all spectral parameters of the 2FGL sources spatially associated with the SNR (three sources within the SNR (Group S), three sources within the Rosette Nebula (Group R), and PSR J0633+0632), the Galactic diffuse emission and the isotropic component, while the integral fluxes of the other point sources are left as free parameters and the spectral indices are fixed to the values reported in 2FGL.

The analyses were performed within a $14^{\circ} \times 14^{\circ}$ square region using 0.1 pixels. The energy range for likelihood analysis is divided into 40 logarithmically spaced energy bins from $0.2 \mathrm{GeV}$ to $300 \mathrm{GeV}$. Figure 2 shows the counts map in the region of interest. We centered the region on the center of the SNR: (R.A., decl.) $=\left(99^{\circ} .75,6^{\circ} .50\right)(\mathrm{J} 2000)$.

\subsection{Morphological Analysis}

For our morphological study, we only used events with energies greater than $0.5 \mathrm{GeV}$ (compared to the $0.2 \mathrm{GeV}$ used in our spectral analysis) to take advantage of the narrower PSF

\footnotetext{
13 As implemented in the publicly available Fermi Science Tools. The documentation concerning the analysis tools and the likelihood fitting procedure is available at http://fermi.gsfc.nasa.gov/ssc/data/analysis/ documentation/Cicerone/.
} 


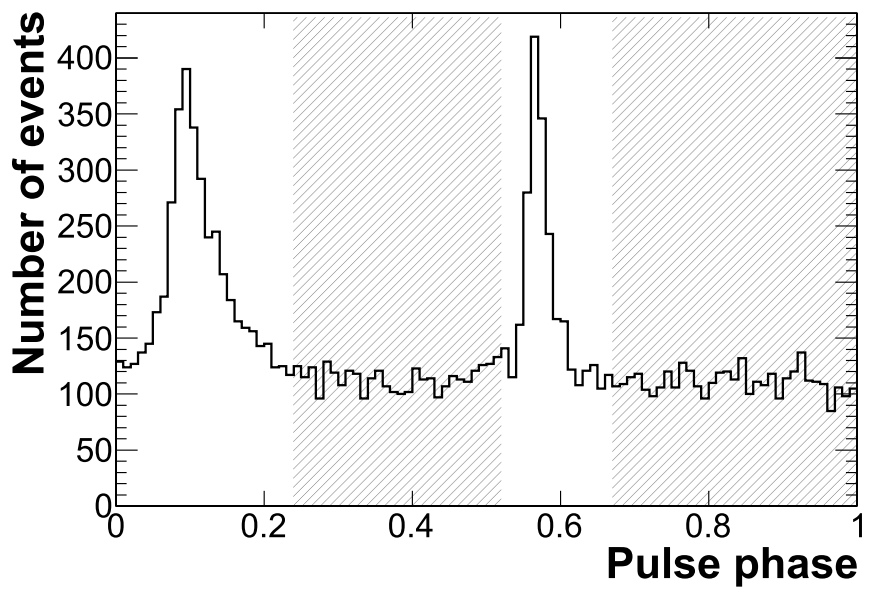

Figure 1. Pulse profile of PSR J0633+0632 using Fermi/LAT data for photon energies of $0.2-300 \mathrm{GeV}$. The off-pulse phase range used in this analysis is shown by shaded regions.

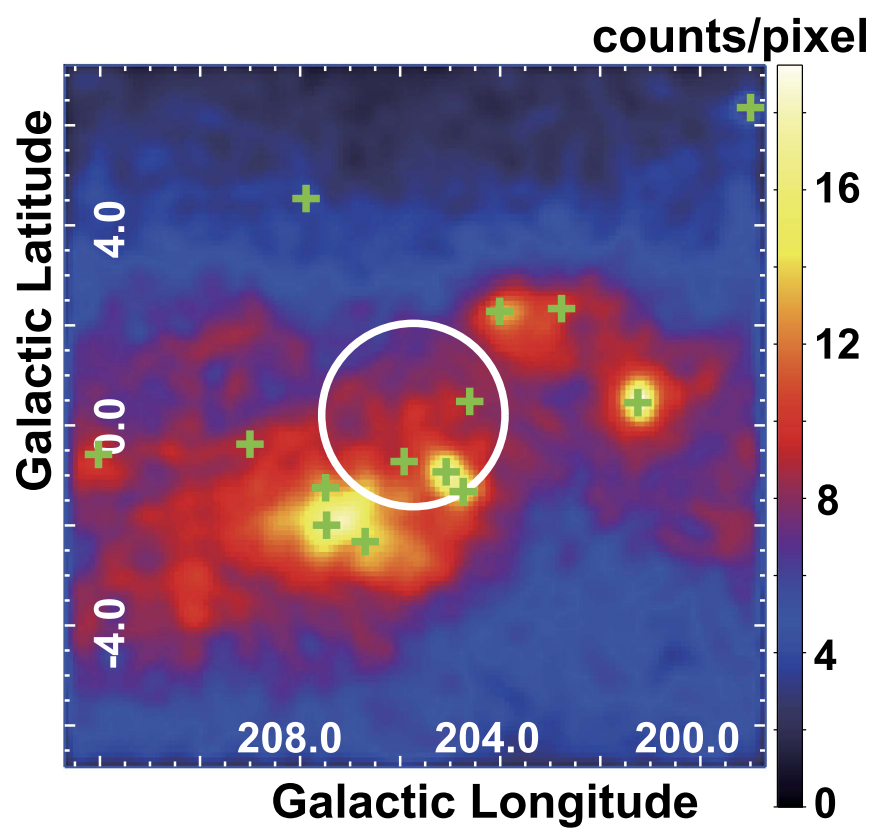

Figure 2. LAT counts map of the region of interest in the $0.5-10 \mathrm{GeV}$ energy range produced using the events during the off-pulse phases of PSR J0633 +0632 . The details of the off-pulse analysis are shown in the text. The counts map is binned using a grid of 0.1 and smoothed with a Gaussian kernel of $\sigma=0.2$. The white circle shows the position of the Monoceros Loop. Crosses indicate the positions of gamma-ray sources listed in the 2FGL catalog (Nolan et al. 2012).

at higher energies. Figure 3 shows the counts map in a $7^{\circ} \times 7^{\circ}$ region centered on the Monoceros Loop, after subtracting the background: the Galactic emission, the isotropic component, and the 2FGL point sources except for the six 2FGL sources in Group $\mathrm{S}$ and $\mathrm{R}$, the parameters of which were the best-fit ones obtained by the likelihood analysis where the emission associated with the SNR and the Rosette Nebula are modeled as the six sources (Model 1 in Table 1). The CO contours overlaid on the map correspond to line intensity integrated over velocities of $0 \mathrm{~km} \mathrm{~s}^{-1}<V<20 \mathrm{~km} \mathrm{~s}^{-1}$ with respect to the local standard of rest, encompassing the velocity of $14 \mathrm{~km} \mathrm{~s}^{-1}$ corresponding to the distance from the Earth $(1.6 \mathrm{kpc}$ assuming the IAU-recommended values $R_{0}=8.5 \mathrm{kpc}$ and $\Theta_{0}=220$ $\mathrm{km} \mathrm{s}^{-1}$ ). The correlation between gamma-rays and the CO line

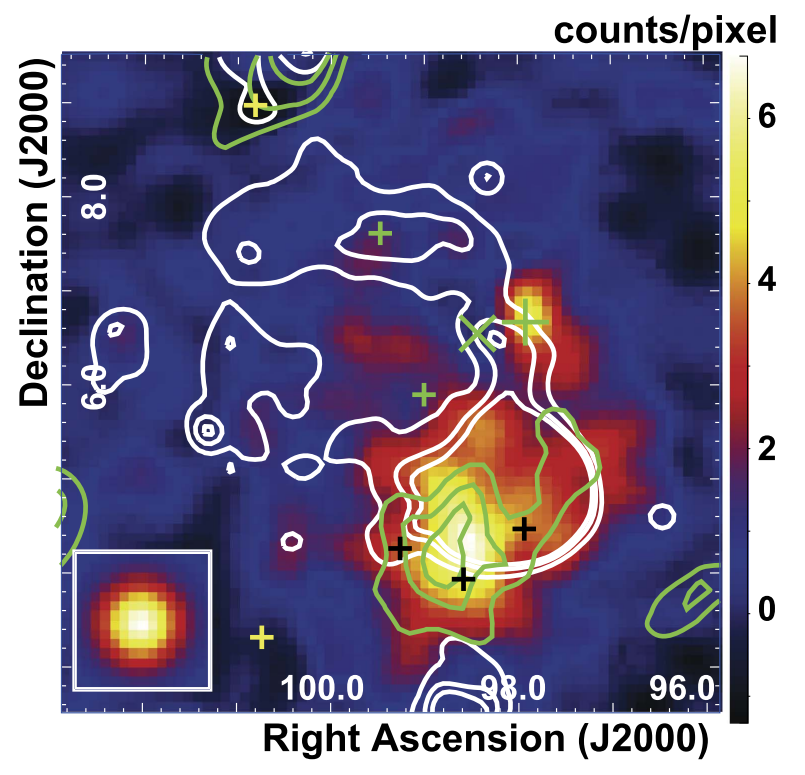

Figure 3. Background-subtracted LAT counts map in the $0.5-10 \mathrm{GeV}$ energy range. The six LAT point sources associated with the Monoceros Loop and the Rosette Nebula, except for PSR J0633+0632, are not included in the background model. The counts map is binned using a grid of 0.1 and smoothed with a Gaussian kernel of $\sigma=0.2$. The inset of the figure shows the simulated LAT PSF with a photon index of 2.5 in the same energy range, adopting the same smoothing. Crosses indicate the positions of gamma-ray sources listed in the 2FGL catalog (Nolan et al. 2012). The green, black, and yellow crosses are for the SNR, the Rosette Nebula, and the others, respectively. The large green cross shows the position of 2FGL J0631.6 +0640 . The green $\mathrm{X}$-mark indicates the position of PSR J0633+0632 (2FGL J0633.7+0633). Green contours correspond to images at ${ }^{12} \mathrm{CO}(J=1 \rightarrow 0)$ line intensities (Dame et al. 2001); contours are at 5, 10, and $15 \mathrm{~K} \mathrm{~km} \mathrm{~s}^{-1}$. White contours are Effelsberg $21 \mathrm{~cm}$ radio continuum (Reich et al. 1997); contours are at 0.4, 0.8, and $1.2 \mathrm{~K}$.

emission around the Rosette Nebula is evident. We note that the LAT standard diffuse model includes this CO emissio$\mathrm{n}$ (Acero et al. 2016a). Thus the residual excess of the gammaray emission indicates that the $\mathrm{CR}$ density in this region is enhanced relative to the surrounding region. The emission north of the $\mathrm{CO}$ region appears point-like and is consistent with the position of the source 2FGL J0631.6+0640.

To evaluate the correlation between the gamma-ray and $\mathrm{CO}$ line emission quantitatively, we fit LAT emission with a spatial template based on the CO line emission for the Rosette Nebula instead of the three 2FGL sources in Group R. We restricted the spatial template to a 2.13 radius about the central cloud (R.A., decl. $)=\left(98^{\circ} .41,4.81\right)(\mathrm{J} 2000)$. Since the edge of the CO emission region is unclear due to statistical noise in the $\mathrm{CO}$ spectral measurements, we introduced the $\mathrm{CO}$ intensity threshold used to create the spatial template as an additional free parameter in the fit. The spectral model was assumed to be a power-law function. The resulting maximum likelihood values with respect to the maximum likelihood for the null hypothesis (no source component associated with the Rosette Nebula and the SNR other than PSR J0633+0632) are summarized in Table 1. The test statistic (TS) value (e.g., Mattox et al. 1996) for the CO image (Model 2 in Table 1) is significantly larger than for all three individual point sources of Group R (Model 1). The threshold value of the CO intensity to 
Table 1

Test Statistics for Different Spatial Models Compared to the Null Hypothesis of a Model with no Source Associated with the Rosette Nebula and the Monoceros Loop $(0.5-300 \mathrm{GeV})$

\begin{tabular}{|c|c|c|}
\hline Model & Test Statistic $^{a}$ & Additional Degrees of Freedom \\
\hline 1: 3 point sources of Group $R+3$ point sources of Group $S^{b}$ & 424.1 & 12 \\
\hline 2: CO image +3 point sources of Group $S$ & 728.8 & $9^{c}$ \\
\hline 3: CO image + Uniform disk + 2FGL J0631.6+0640 ${ }^{\mathrm{d}}$ & 937.6 & 10 \\
\hline 5: CO image + Radio template ${ }^{\mathrm{e}}+2$ FGL J0631.6+0640 & 922.2 & 7 \\
\hline
\end{tabular}

Notes.

a $-2 \ln \left(L_{0} / L\right)$, where $L$ and $L_{0}$ are the maximum likelihoods for the model with/without the source component, respectively. The model for $L_{0}$ includes PSR J0633 +0632 .

${ }^{\mathrm{b}}$ The three sources in the 2FGL source list associated with the Rosette Nebula (Nolan et al. 2012) are referred to as Group R in the text. The three sources listed in the 2FGL source list associated with the Monoceros Loop are referred to as Group S in the text. 2FGL J0633.7+0633 (PSR J0633+0632) is not included in them.

${ }^{\mathrm{c}}$ The additional degrees of freedom for the $\mathrm{CO}$ image is two for the spectral shape and one for the analysis threshold to extract emission. The details are shown in the text.

d 2 FGL J0631.6+0640 is included in Group S.

e The radio template was obtained from $408 \mathrm{MHz}$ radio data (Taylor et al. 2003) by excluding the region around the Rosette Nebula, where the emission is predominantly thermal. The additional degrees of freedom for the radio template are two for the spectral shape (a power law).

maximize the likelihood value is $0 \mathrm{~K} \mathrm{~km} \mathrm{~s}^{-1}$, where the parameter varies from 0 to $4 \mathrm{~K} \mathrm{~km} \mathrm{~s}^{-1}$.

We further characterized the morphology of gamma-ray emission associated with the Monoceros Loop. Figure 4 shows the counts map in a $7^{\circ} \times 7^{\circ}$ region centered on the SNR, after subtracting the background and the emission from the Rosette Nebula. The background here consists of the Galactic emission, the isotropic component, the $\mathrm{CO}$ spatial template, 2FGL J0631.6+0640 in Group S, and the 2FGL point sources except for the other two sources in Group S (2FGL J0636.0 +0554 and 2FGL J0637.8+0737), the parameters of which were the best-fit ones obtained by the likelihood analysis with the CO template plus all three point sources of Group S (Model 2 in Table 1). A spatially extended emission region within the SNR becomes apparent.

To quantitatively evaluate the detection significance of the spatially extended emission, we tested the hypothesis of an extended emission region inside the Monoceros Loop against the model with the two individual point sources in Group S (2FGL J0636.0+0554 and 2FGL J0637.8+0737). We fitted the LAT counts by replacing all three point sources of Group $S$ by one point source (2FGL J0631.6+0640 in Group S) and a spatially extended component modeled as either a uniform disk or a Gaussian emission profile (Model 3 and 4 in Table 1). The spectral shapes for both additional sources were assumed to be power-law functions. We varied the radius ( $1 \sigma$ for a Gaussian profile) and location of the extended components and evaluated the maximum likelihood values. The resulting maximum likelihood values with respect to the maximum likelihood for the null hypothesis are summarized in Table 1 . The TS values for the uniform disk or the Gaussian profile plus the point source and the CO template (Model 3 and 4) are much larger than for the three 2FGL sources plus the CO template (Model 2) albeit having fewer degrees of freedom. The Gaussian profile (Model 4) provides a greater likelihood than the uniform disk (Model 3): the TS value increases by 26.7. The maximum likelihood Gaussian profile has a radius $(\sigma)$ of $2.3_{-0.5}^{+0.6}$ centered on (R.A., decl. $)=\left(99^{\circ} .86,6^{\circ} .93\right)(\mathrm{J} 2000)$. The error of the centroid is 0.35 at a $68 \%$ confidence level. The detection significances for the best-fit Gaussian profile and 2FGL J0631.6+0640 at energies of $>0.5 \mathrm{GeV}$ are $\sim 14 \sigma$ and $\sim 11 \sigma$, respectively. We note that if we add the five eliminated

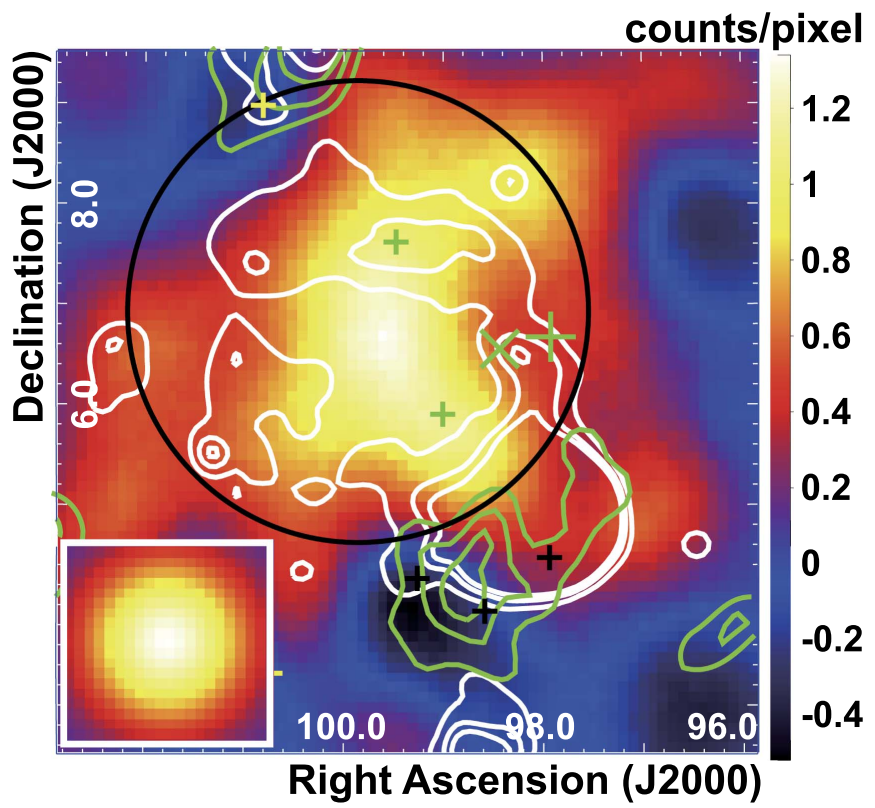

Figure 4. Background-subtracted LAT counts map in the $0.5-10 \mathrm{GeV}$ energy range. The emission predicted from the $\mathrm{CO}$ spatial template for the Rosette Nebula is subtracted, whereas the two 2FGL point sources associated with the SNR (2FGL J0636.0+0554, 2FGL J0637.8+0737) are not included in the background model. The counts map is binned using a grid of 0.1 and smoothed with a Gaussian kernel of $\sigma=0.5$. The black circle shows the best-fit Gaussian spatial model ( $1 \sigma$ radius). The details of the overlays are described in the caption of Figure 3.

2FGL sources on top of the best-fit model, the TS value increases by only 6.2 for 10 additional degrees of freedom, i.e., there is no statistical evidence for the presence of these sources in addition to the extended templates. Also, we note that the maximum likelihood value for a $408 \mathrm{MHz}$ radio template with suppression of emission from Rosette Nebula (Model 5) was significantly worse than the best-fit Gaussian model, indicating that the gamma-ray emission around the Monoceros Loop is not strongly spatially associated with the shock region of the SNR as traced by radio. Finally, we examined the residual map after fitting as shown in Figure 5. There is no prominent gamma-ray emission left in the map. Therefore, we adopted the 


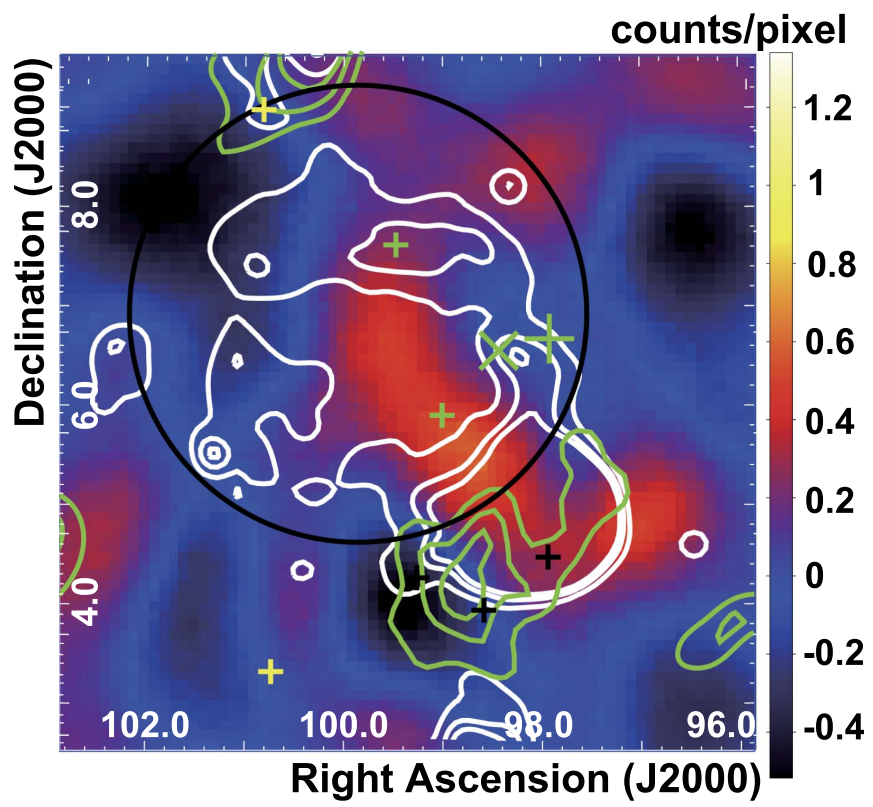

Figure 5. Background-subtracted LAT counts map in the $0.5-10 \mathrm{GeV}$ energy range. Here, the background model is the best-fit spatial model described in the text. The details of the binning and smoothing are the same as Figure 4. The scale of the color bar is also the same as Figure 4.

Gaussian template with maximum likelihood parameters for the whole SNR in the following spectral analysis.

\subsection{Spectral Analysis}

To measure the spectra of the SNR and the Rosette Nebula, we used a maximum likelihood fit using the best-fit spatial model over the energy range from 0.2 to $300 \mathrm{GeV}$. Figures 6 and 7 show the resulting spectral energy distributions (SEDs) for the SNR and the Rosette Nebula, respectively. If the detection is not significant in an energy bin, i.e., the improvement of the TS value with respect to the null hypothesis is less than four (corresponding to $2 \sigma$ for one additional degree of freedom) then we calculated a $90 \%$ confidence level upper limit assuming a photon index of two.

At least three different sources of systematic uncertainties affect our analysis: uncertainties in the LAT event selection efficiency, the adopted diffuse model and the morphological templates. Uncertainties in the LAT effective area were evaluated by comparing the efficiencies of analysis cuts for data and simulation of observations of Vela and the limb of the Earth, among other consistency checks (Ackermann et al. 2012). For P7REP_SOURCE_V15, these studies suggest a $10 \%$ systematic uncertainty below $100 \mathrm{MeV}$, decreasing linearly with the logarithm of energy to $5 \%$ in the range between $316 \mathrm{MeV}$ and $10 \mathrm{GeV}$ and increasing linearly with the logarithm of energy up to $15 \%$ at $1 \mathrm{TeV}$. We adopted the strategy described in Ackermann et al. (2013) and Acero et al. (2016b) to evaluate the systematic uncertainties due to the modeling of interstellar emission. Results obtained using the standard model in Section 3.2 were compared with the results of eight alternative interstellar emission models. These models were created by varying the uniform spin temperature used to estimate the column densities of interstellar atomic hydrogen, the vertical height of the CR propagation halo, and the CR source distribution in the Galaxy.

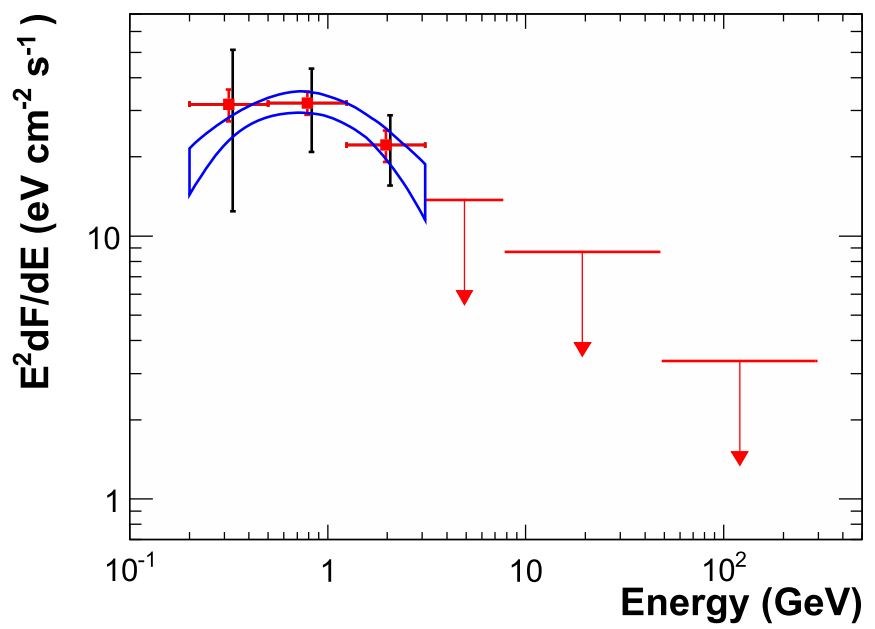

Figure 6. Spectral energy distribution of gamma-ray emission toward the Monoceros Loop. The measured LAT fluxes are shown as red squares with horizontal lines indicating the energy range. Statistical and systematic error bars are shown in red and black, respectively. Flux upper limits at the $90 \%$ confidence level are shown for energy bins when the detection was not significant (test statistic $<4$ ). The blue region is the $68 \%$ confidence range (no systematic error) of the LAT spectrum assuming that the spectral shape is a log parabola.

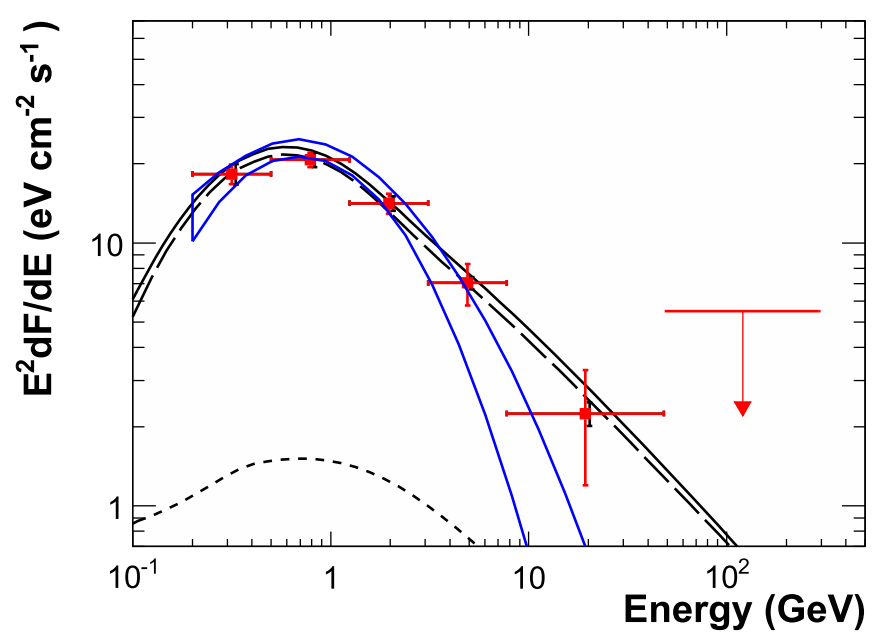

Figure 7. Spectral energy distribution of the gamma-ray emission measured by the LAT for the Rosette Nebula and the spectrum expected from the model discussed in the text. The details of the LAT spectrum and the modeled emission processes are described in the captions of Figures 6 and 8, respectively.

We similarly gauged the uncertainties due to the morphological template by comparing the results with those obtained by changing the radius of the Gaussian template within its $\pm 1 \sigma$ error. The total systematic errors were set by adding the above uncertainties in quadrature. If the total systematic error in an energy bin was $>100 \%$, the point was replaced by an upper limit. This is relevant for the fourth energy bin $(3.105-7.746 \mathrm{GeV})$ in Figure 6 where an upper limit is presented due to the large systematic error, though the TS value is $\sim 9$. The dominant systematic error for the measurement of the SNR spectrum arises from the uncertainty of the diffuse model below $0.5 \mathrm{GeV}$ and the morphological uncertainty above $0.5 \mathrm{GeV}$, respectively.

We searched for a spectral break in the LAT energy range by comparing the likelihood values of a spectral fit over the whole energy range considered based on a simple power law and a log 
Table 2

Test Statistics and Parameters for Spectral Models (0.2-300 GeV)

\begin{tabular}{lccc}
\hline \hline $\begin{array}{l}\text { Spectral } \\
\text { Model }\end{array}$ & $\begin{array}{c}\text { Test } \\
\text { Statistic }^{\mathrm{a}}\end{array}$ & $\begin{array}{c}\text { Additional } \\
\text { Degrees of } \\
\text { Freedom }\end{array}$ & Spectral Parameters \\
\hline $\begin{array}{l}\text { Monoceros } \\
\text { Loop }\end{array}$ & & & \\
\hline Power Law & 0 & 2 & $E^{-p} ; p=2.27 \pm 0.03$ \\
\hline Log Parabola & 51 & 3 & $\left(\frac{E}{1 \mathrm{GeV}}\right)^{-p_{1}-p_{2} \log \left(\frac{E}{1 \mathrm{GeV}}\right)}$ \\
& & $p_{1}=2.23 \pm 0.06$ \\
$p_{2}=0.35 \pm 0.03$
\end{tabular}

Rosette

Nebula

\begin{tabular}{lccc}
\hline Power Law & 0 & 2 & $E^{-p} ; p=2.32 \pm 0.02$ \\
\hline Log Parabola & 88 & 3 & $\left(\frac{E}{1 \mathrm{GeV}}\right)^{-p_{1}-p_{2} \log \left(\frac{E}{1 \mathrm{GeV}}\right)}$ \\
& & $p_{1}=2.29 \pm 0.03$ \\
& & $p_{2}=0.39 \pm 0.02$ \\
\hline
\end{tabular}

Note.

a $-2 \ln \left(L_{0} / L\right)$, where $L$ and $L_{0}$ are the maximum likelihood values for the model under consideration and the power-law model, respectively.

parabola function. TS values and best-fit parameters are summarized in Table 2. The values for a log parabola function correspond to improvements at the $>6 \sigma$ confidence level for the SNR and $>9 \sigma$ for the Rosette Nebula when only statistical uncertainties are taken into account. For the SNR, we further investigated the systematic effects on the above spectral analysis. Accounting for systematics in the fit, the curved shape is still preferred over a power law at a confidence level $>5 \sigma$. By comparing the spectral parameters of a log parabola function for both sources, the spectral shapes are consistent within the statistical errors at our current sensitivity. Assuming the spectral shape is a log parabola function, the gamma-ray luminosities integrated over the energy range $0.2-300 \mathrm{GeV}$ inferred from our analysis are $\sim 4 \times 10^{34} \mathrm{erg} \mathrm{s}^{-1}$ for the SNR and $\sim 3 \times 10^{34} \mathrm{erg} \mathrm{s}^{-1}$ for the Rosette Nebula, respectively.

\section{DISCUSSION}

An extended region of gamma-ray emission was found to be spatially coincident with the Monoceros SNR by Acero et al. (2016b). We confirmed the extended emission with this more detailed analysis. Since no pulsar wind nebula has been discovered so far within the SNR (e.g., Roberts 2004), the likely explanation for the bulk of this gamma-ray emission is the interaction of high-energy particles accelerated in the shocks of the Monoceros Loop with ambient interstellar matter and radiation fields. The morphological difference between the gamma-ray emission and the radio emission can be explained by the inhomogeneity of the nearby gas, which is irradiated by the accelerated CRs that have escaped from the shocked regions. This hypothesis would also readily explain the enhanced emission from the nearby Rosette Nebula where the same population of high-energy particles would produce a bright gamma-ray signal when interacting in the dense molecular clouds traced by the $\mathrm{CO}$ emission. We note that the possibility of a pulsar wind nebula without detectable radio emission cannot be ruled out for the explanation of the enhanced emission around the SNR. Also, we cannot rule out that some of the emission around the SNR is produced by dark gas, i.e., gas that is not accounted for in $\mathrm{HI}$ or $\mathrm{CO}$ surveys. Its distribution cannot be modeled precisely, yet large quantities of dark gas have been found surrounding nearby molecular clouds (Grenier et al. 2005). In contrast, it is difficult to explain the enhanced emission around the Rosette Nebula only by dark gas considering a good fit of the CO template and the feature of dark gas that is mostly at the outskirt of the cloud.

Broadband emission from the Monoceros Loop SNR was modeled under the assumption that gamma-rays are emitted by a population of accelerated protons and electrons. We assumed that relativistic electrons and protons have the same injection spectrum and occupy the same spatial volume characterized by a constant magnetic field strength and matter density. We used the following equation to model the momentum distribution of injected particles:

$$
Q_{e, p}(p)=a_{e, p}\left(\frac{p}{1 \mathrm{GeV} c^{-1}}\right)^{-s_{\mathrm{L}}}\left\{1+\left(\frac{p}{p_{\mathrm{br}}}\right)^{2}\right\}^{-\left(s_{\mathrm{H}}-s_{\mathrm{L}}\right) / 2},
$$

where $p_{\mathrm{br}}$ is the break momentum, $s_{\mathrm{L}}$ is the spectral index below the break and $s_{\mathrm{H}}$ above the break. $a_{e, p}$ are normalizations for the electron and proton components, respectively. Because the details of the proton/electron injection process are poorly known, we adopt a minimum momentum of $100 \mathrm{MeV} c^{-1}$.

Electrons suffer energy losses due to ionization, Coulomb scattering, bremsstrahlung, synchrotron emission, and inverse Compton (IC) scattering. The evolution of the momenta spectra $N_{e, p}(p, t)$ are calculated from the following equation:

$$
\frac{\partial N_{e, p}}{\partial t}=\frac{\partial}{\partial p}\left(b_{e, p} N_{e, p}\right)+Q_{e, p}
$$

where $b_{e, p}=-d p / d t$ is the momentum loss rate and $Q_{e, p}$ is the particle injection rate. We assumed that the shock produced particles at a constant rate, so $Q_{e, p}$ is constant. To derive the gamma-ray emission spectrum, we calculated $N_{e, p}\left(p, T_{0}\right)$ numerically, where $T_{0}$ is the SNR age of $3 \times 10^{4}$ years. Momentum losses for protons are neglected because the timescale for radiative losses via neutral pion production is $\sim 10^{7} /\left(\bar{n}_{\mathrm{H}} / 1 \mathrm{~cm}^{-3}\right)$ years, where $\bar{n}_{\mathrm{H}}$ is the gas density averaged over the volume occupied by high-energy particles. Gammaray emission by secondary leptons produced from charged pion decay was neglected. Generally, this is a negligible contribution unless the gas density is comparable to that in dense molecular clouds and the SNR has reached the later stages of its evolution, or the injected electron-to-proton ratio is much lower than locally observed. The calculation of the spectrum of $\pi^{0}$ decay gamma-rays from interactions between protons and ambient hydrogen was adopted from Dermer (1986). A scaling factor of 1.84 accounted for helium and heavier nuclei in target material and CRs (Mori 2009). Contributions from bremsstrahlung and IC scattering by accelerated electrons are computed based on Blumenthal \& Gould (1970), and synchrotron radiation is evaluated using the work of Crusius \& Schlickeiser (1986).

First, we considered a model with the Monoceros Loop SNR dominated by $\pi^{0}$-decay. The gamma-ray spectrum constrains the number index of accelerated protons to be $s_{\mathrm{H}} \approx 2.8$ in the 
Table 3

Model Parameters for the Monoceros Loop

\begin{tabular}{lccccccc}
\hline \hline Model & $K_{\mathrm{ep}}{ }^{\mathrm{a}}$ & $s_{\mathrm{L}}{ }^{\mathrm{b}}$ & $\begin{array}{c}p_{\mathrm{br}}{ }^{\mathrm{c}} \\
\left(\mathrm{GeV} c^{-1}\right)\end{array}$ & $s_{\mathrm{H}}{ }^{\mathrm{d}}$ & $\begin{array}{c}B \\
(\mu \mathrm{G})\end{array}$ & $\begin{array}{c}\bar{n}_{\mathrm{H}}{ }^{\mathrm{e}} \\
\left(\mathrm{cm}^{-3}\right)\end{array}$ & $\begin{array}{c}W_{p}{ }^{\mathrm{f}} \\
\left(10^{49} \mathrm{erg}\right)\end{array}$ \\
\hline (a) Pion & 0.01 & 1.5 & 2.0 & 2.8 & 35 & 3.6 & 7.6 \\
(b) Bremsstrahlung & 1 & 1.5 & 1.0 & 3.0 & 9 & 3.6 & 0.98 \\
(c) Inverse Compton & 1 & 1.5 & 20 & 4.0 & 1.1 & 0.01 & 1.4 \\
\hline
\end{tabular}

Notes.

The ratio electrons-to-protons at $1 \mathrm{GeV} c^{-1}$.

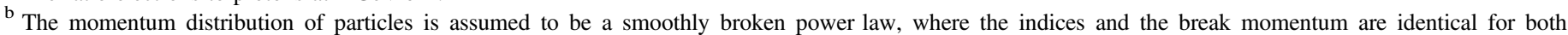
accelerated protons and electrons. $s_{\mathrm{L}}$ is the spectral index in momentum below the break.

${ }^{c} p_{\text {br }}$ is the break momentum.

d Spectral index in momentum above the break.

e Average hydrogen number density of ambient medium.

${ }^{\mathrm{f}}$ The distance from the Earth is assumed to be $1.6 \mathrm{kpc}$ (Turner 1976; Odegard 1986). The total energy is calculated for particles $>100 \mathrm{MeV} c^{-1}$.

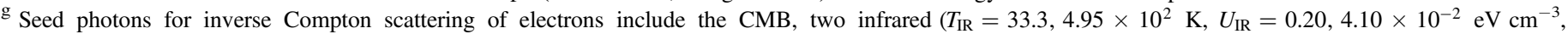
respectively), and two optical components $\left(T_{\mathrm{opt}}=3.72 \times 10^{3}, 1.11 \times 10^{4} \mathrm{~K}, U_{\mathrm{opt}}=0.30,0.11 \mathrm{eV}^{-3}\right.$, respectively) in the vicinity of the Monoceros Loop.

high-energy regime. We adopted a spectral index of $s_{\mathrm{L}}=1.5$ to explain the radio continuum spectrum (Xiao \& Zhu 2012). We note that the radio spectrum was estimated from the full SNR with the exception of the Rosette Nebula region that is dominated by strong thermal emission. Since we expect curvature in the $\mathrm{GeV}$ energy band due to the kinematics of $\pi^{0}$ production and decay, it is difficult to constrain a break in the proton momenta spectrum from the gamma-ray spectrum. The gamma-ray spectrum thus provides only an upper bound to the momentum break at $\sim 10 \mathrm{GeV} c^{-1}$. We adopt a break at the best-fit value, $2 \mathrm{GeV} \mathrm{c} c^{-1}$. The density is fixed to $3.6 \mathrm{~cm}^{-3}$ based on the HI observations (Xiao \& Zhu 2012). The resulting total proton energy, $W_{p} \sim 7.6 \times 10^{49} \cdot\left(3.6 \mathrm{~cm}^{-3} / \bar{n}_{\mathrm{H}}\right)$. $(d / 1.6 \mathrm{kpc})^{2} \mathrm{erg}$, is less than $10 \%$ of the typical kinetic energy of a supernova explosion. For the electron-to-proton ratio measured at Earth, $K_{\mathrm{ep}} \equiv a_{e} / a_{p}=0.01$, the magnetic field strength is determined to be $B \sim 35 \mu \mathrm{G}$ by the radio data. Using these model parameters (Table 3), we obtained the SEDs shown in Figure 8(a).

In the case of leptonic scenarios, we assume $K_{\text {ep }}=1$ to produce the gamma-ray emission predominantly from the electrons. The radio spectrum (Xiao \& Zhu 2012) is difficult to be modeled as the synchrotron radiation when we fit the gamma-ray spectrum with a model dominated by electron bremsstrahlung, as shown in Figure 8(b).

The other leptonic scenario is an IC-dominated model. IC gamma-rays originate from the interaction of high-energy electrons with the cosmic microwave background (CMB) as well as optical and infrared radiation fields. Galactic radiation fields were adopted from Porter et al. (2008) at the location of the Monoceros Loop. These very complex spectra are approximated by two infrared and two optical blackbody components. It is hard to reproduce the multi-wavelength spectrum well with an IC-dominated model shown in Figure 8(c). In addition, the ratio between IC and synchrotron fluxes constrained the magnetic field to be less than $\sim 2 \mu \mathrm{G}$ and requires a low gas density of $\bar{n}_{\mathrm{H}} \sim 0.01 \mathrm{~cm}^{-3}$ to suppress the electron bremsstrahlung, which is unlikely.

In conclusion, the bulk of the gamma-ray emission from the Monoceros SNR is most likely from $\pi^{0}$ decay produced by the interactions of protons with ambient hydrogen. It is then reasonable to explain the gamma-ray spectrum of the Rosette Nebula by the same process. If the protons are accelerated in

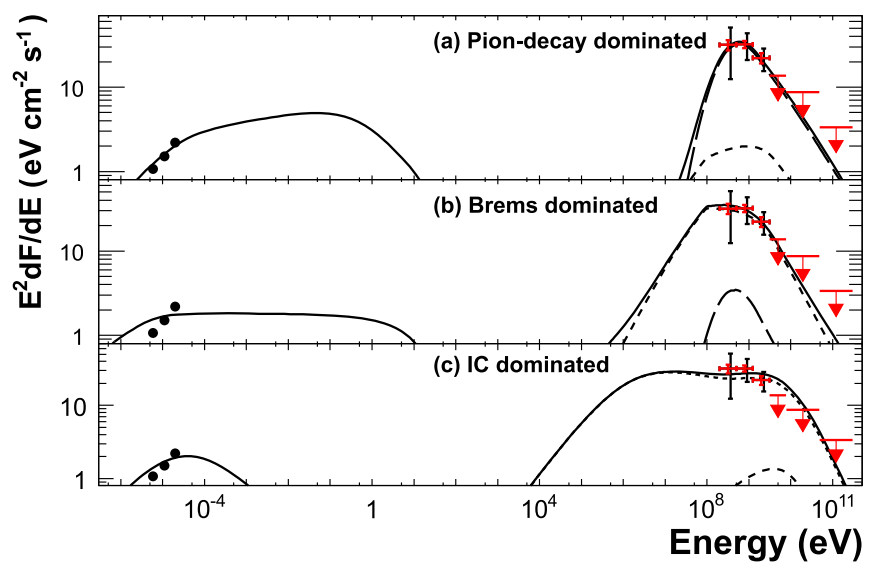

Figure 8. Multi-band spectrum of the Monoceros Loop. LAT measurements reported in Figure 6 are shown alongside radio continuum measurements (Xiao \& Zhu 2012). Radio emission is modeled as synchrotron radiation, while gamma-ray emission is modeled by different combinations of $\pi^{0}$-decay (longdashed curve), bremsstrahlung (dashed curve), and inverse Compton (IC) scattering (dotted curve). As described in the text, the models are (a) $\pi^{0}$-decay dominated, (b) bremsstrahlung dominated, and (c) IC-dominated.

the whole SNR in the same manner and are not strongly affected by spectral deformation due to CR diffusion processes, the shape of the proton spectrum in the Rosette Nebula is expected to be the same as in the Monoceros Loop. Figure 7 shows the gamma-ray spectrum of the Rosette Nebula with the $\pi^{0}$-decay dominated model assuming the density in the molecular clouds is $100 \mathrm{~cm}^{-3}$. The spectrum can be reproduced without any change from the proton momentum spectrum of the SNR. The resulting total proton energy, $W_{p} \sim 0.18 \times 10^{49} \cdot\left(100 \mathrm{~cm}^{-3} / \bar{n}_{\mathrm{H}}\right) \cdot(d / 1.6 \mathrm{kpc})^{2} \mathrm{erg}$, is about $2 \%$ of that for the $\pi^{0}$-decay model of the SNR, which is reasonable considering the solid angle of the Rosette Nebula with respect to the SNR and uncertainty of the matter density. We note that these CR energies for the Monoceros Loop SNR and the Rosette Nebula are the enhancements of the CR density in addition to that implicit in the standard Galactic diffuse emission model.

To summarize, the assumption that the gamma-ray emission from the Monoceros SNR is dominated by decay of $\pi^{0}$ produced in nucleon-nucleon interactions of hadronic CRs with interstellar matter is a natural scenario that can also readily 
explain the emission from the nearby Rosette Nebula as interactions of the same population of CRs in the dense molecular cloud. Similarly to SNR HB 3 (Katagiri et al. 2016), it should be emphasized that our observations toward the Monoceros Loop provide a rare and valuable example for which the emissions from both the SNR and the interacting molecular clouds are detected.

\section{CONCLUSIONS}

We analyzed gamma-ray measurements by the LAT in the region of the Monoceros Loop. The brightest gamma-ray peak is spatially correlated with the Rosette Nebula. A template derived from the $\mathrm{CO}$ gas distribution fits the morphology of the gamma-ray emission better than a set of individual point sources. Gamma-ray emission from an extended source was also found inside the Monoceros Loop. A Gaussian emission profile of 2.3 radius $(1 \sigma)$ is a substantially better match to the gamma-ray data when compared to the 2FGL sources J0636.0 +0554 and J0637.8+0737. The gamma-ray spectra of both extended components were significantly better reproduced by a curved shape than a simple power law.

Their respective luminosities integrated over the energy range of $0.2-300 \mathrm{GeV}$ are $\sim 4 \times 10^{34} \mathrm{erg} \mathrm{s}^{-1}$ for the SNR and $\sim 3 \times 10^{34} \mathrm{erg} \mathrm{s}^{-1}$ for the Rosette Nebula. The decay of $\pi^{0}$ produced by interactions of hadrons accelerated by the remnant with interstellar gas can naturally explain the gamma-ray emission of the remnant and that of the nebula.

The Fermi/LAT Collaboration acknowledges generous ongoing support from a number of agencies and institutes that have supported both the development and the operation of the LAT as well as scientific data analysis. These include the National Aeronautics and Space Administration and the Department of Energy in the United States, the Commissariat à l'Energie Atomique and the Centre National de la Recherche Scientifique/Institut National de Physique Nucléaire et de Physique des Particules in France, the Agenzia Spaziale Italiana and the Istituto Nazionale di Fisica Nucleare in Italy, the Ministry of Education, Culture, Sports, Science and Technology (MEXT), High Energy Accelerator Research Organization (KEK) and Japan Aerospace Exploration Agency (JAXA) in Japan, and the KA Wallenberg Foundation, the Swedish Research Council and the Swedish National Space Board in Sweden.

Additional support for science analysis during the operations phase is gratefully acknowledged from the Istituto Nazionale di Astrofisica in Italy and the Centre National d'Études Spatiales in France.
We thank Luigi Tibaldo for helpful comments and discussions on the dark gas.

\section{REFERENCES}

Abdo, A. A., Ackermann, M., Ajello, M., et al. (The Fermi LAT Collaboration) 2009, APh, 32, 193

Abdo, A. A., Ajello, M., Allafort, A., et al. (The Fermi LAT Collaboration) 2013, ApJS, 208, 17

Acciari, V. A., Aliu, E., Arlen, T., et al. 2009, ApJL, 698, L94

Acero, F., Ackermann, M., Ajello, M., et al. (The Fermi LAT Collaboration) 2016a, ApJS, 223, 26

Acero, F., Ackermann, M., Ajello, M., et al. (The Fermi LAT Collaboration) 2016b, ApJS, 224, 8

Ackermann, M., Ajello, M., Albert, A., et al. (The Fermi LAT Collaboration) 2012, ApJS, 203, 4

Ackermann, M., Ajello, M., Allafort, A., et al. (The Fermi LAT Collaboration) 2013, Sci, 339, 807

Aharonian, F. A., Akhperjanian, A. G., Bazer-Bachi, A. R., et al. 2007, A\&A, 469, L1

Atwood, W. B., Abdo, A. A., Ackermann, M., et al. (The Fermi LAT Collaboration) 2009, ApJ, 697, 1071

Blandford, R. D., \& Eichler, D. 1987, PhR, 154, 1

Blumenthal, G. R., \& Gould, R. J. 1970, RvMP, 42, 237

Bregeon, J., Charles, E., Wood, M. \& The Fermi LAT Collaboration 2013, arXiv: 1304.5456

Caliandro, G. A., Hill, A. B., Torres, D. F., et al. 2013, MNRAS, 436, 740

Cheung, C. C. \& The Fermi LAT Collaboration 2013, arXiv:1304.3475

Crusius, A., \& Schlickeiser, R. 1986, A\&A, 164, L16

Dame, T. M., Hartmann, D., \& Thaddeus, P. 2001, ApJ, 547, 792

Dermer, C. D. 1986, A\&A, 157, 223

Fountain, W. F., Gary, G. A., \& Odell, C. R. 1979, ApJ, 229, 971

Grenier, I. A., Casandjian, J.-M., \& Terrier, R. 2005, Sci, 307, 1292

Hartman, R. C., Bertsch, D. L., Bloom, S. D., et al. 1999, ApJS, 123, 79

Hobbs, G. B., Edwards, R. T., \& Manchester, R. N. 2006, MNRAS, 369, 655

Katagiri, H., Tibaldo, L., Ballet, J., et al. 2011, ApJ, 741, 44

Katagiri, H., Yoshida, K., Ballet, J., et al. 2016, ApJ, 818, 114

Kerr, M., Ray, P. S., Johnston, S., Shannon, R. M., \& Camilo, F. 2015, ApJ, 814,128

Kocevski, D., Ohno, M., Racusin, J., et al. 2013, GCN, 14574, 1

Leahy, D. A., Naranan, S., \& Singh, K. P. 1986, MNRAS, 220, 501

Mattox, J. R., Bertsch, D. L., Chiang, J., et al. 1996, ApJ, 461, 396

Mori, M. 2009, APh, 31, 341

Nolan, P. L., Abdo, A. A., Ackermann, M., et al. (The Fermi LAT Collaboration) 2012, ApJS, 199, 31

Odegard, N. 1986, ApJ, 301, 813

Porter, T. A., Moskalenko, I. V., Strong, A. W., Orlando, E., \& Bouchet, L. 2008, ApJ, 682, 400

Reich, P., Reich, W., \& Furst, E. 1997, A\&As, 126, 413

Roberts, M. S. E. 2004, The Pulsar Wind Nebula Catalog (March 2005 version) (Montreal, Quebec, Canada: McGill Univ) (http://www.physics.mcgill.ca/ pulsar/pwncat.html)

Taylor, A. R., Gibson, S. J., Peracaula, M., et al. 2003, AJ, 125, 3145

Thompson, D. J., Baldini, L., \& Uchiyama, Y. 2012, APh, 39, 22

Turner, D. G. 1976, ApJ, 210, 65

Xiao, L., \& Zhu, M. 2012, A\&A, 545, A86 